\section{Dr. Chira replies}

\section{To the Editor:}

We appreciate the contribution of Dr. Chanchlani, et al regarding their retrospective assessment of transfer of adolescent rheumatology patients in the United Kingdom because it demonstrated the real-world challenges of implementing a successful transition program ${ }^{1}$. The UK has always been a leader in rheumatology adolescent transitional care as noted by this group's previous work ${ }^{2}$. While their recent data showed favorable results in actual transfers, as noted by adult rheumatology referral notes being sent to the pediatric rheumatology consultant $(86 \%)$, concerns were raised with regard to the timeliness of visits (mean time, 115 days) and establishing continuity. Studies have shown that the lack of established care with an adult provider can increase problems of morbidity, mortality, and healthcare costs for young people with childhood-onset conditions, including rheumatic diseases, and we need tools to improve these outcomes by ensuring successful transition and transfer ${ }^{3}$.

Creation of a medical summary is an important component of transition because it is an instrument that can bridge the medical communities and can actively engage the patients to better understand and articulate their condition and its effect on their lives. Unfortunately, a medical summary is not routinely done in conjunction with the patient and family, as noted by the Childhood Arthritis and Rheumatology Research Alliance (CARRA) survey of North American pediatric rheumatologists that showed $16 \%$ of respondents providing this service ${ }^{4}$. Even when a summary is created by healthcare providers for one another, it is not always shared with the patients (only about $41 \%$ for this UK patient cohort received a copy) ${ }^{1}$, limiting its utility in patient education and engagement. Electronic medical records (which may include patient portals) are an opportunity to improve informational continuity, but we must not discount the importance of interpersonal interactions and the team approach to make these transfers successful.

In the United States, limited resources prevent having a dedicated transition program at every pediatric rheumatology center. We look to other countries, such as the United Kingdom and Canada, which have a more established history of studying and addressing the challenges, but realize that structural differences in those healthcare systems likely play a significant role. The "Six Core Elements of Health Care Transition" is an adaptable resource that outlines methods and key issues that can and should be addressed by all providers and others involved in the process, regardless of the healthcare system structure ${ }^{5}$. Its recent revision strongly advocates for establishing a written transition policy that includes input and agreement of adult care providers with their pediatric colleagues. More research needs to be done to train providers about these concepts, including how to integrate this process into their workflow, and to study the effect on longterm patient outcomes.

PETER CHIRA, MD, MS, Clinical Associate Professor of Pediatrics, University of California, and Rady Children's Hospital, San Diego, California, USA. Address correspondence to Dr. P. Chira, 3020 Children's Way, Mail Code 5113, San Diego, California 92123, USA.

E-mail: pchira@ucsd.edu

\section{REFERENCES}

1. Chanchlani N, McDonagh JE, McGee M. Informational continuity is integral for successful transition of adolescents. J Rheumatol 2015;42:901-2.

2. McDonagh JE, Shaw KL, Southwood TR. Growing up and moving on in rheumatology: development and preliminary evaluation of a transitional care programme for a multicentre cohort of adolescents with juvenile idiopathic arthritis. J Child Health Care 2006;10:22-42.

3. Okumura MJ, Hersh AO, Hilton JF, Lotstein DS. Change in health status and access to care in young adults with special health care needs: results from the 2007 national survey of adult transition and health. J Adolesc Health 2013;52:413-8.

4. Chira P, Ronis T, Ardoin S, White P. Transitioning youth with rheumatic conditions: perspectives of pediatric rheumatology providers in the United States and Canada. J Rheumatol 2014;41:768-79.

5. Got Transition. [Internet. Accessed February 4, 2015.] Available from: www.gottransition.org

J Rheumatol 2015;42:5; doi:10.3899/jrheum.141579 